\title{
O063: Healthcare-associated bloodstream infections in Finland, 1999-2011 - adjusted ranking of hospitals by Staphylococcus aureus rates
}

\author{
T Kärki , J Ollgren, O Lyytikäinen
}

From 2nd International Conference on Prevention and Infection Control (ICPIC 2013)

Geneva, Switzerland. 25-28 June 2013

\section{Introduction}

Bloodstream infections (BSI), including those caused by Staphylococcus aureus (SA-BSI) are often severe. Relatively large proportion of SA-BSIs are preventable, and their rate has been used as an indicator for hospital performance in infection control.

\section{Objectives}

The objective of this study was to analyze the Finnish surveillance data in order to assess hospital rankings according to crude and adjusted rates of overall BSI and SA-BSI.

\section{Methods}

11 Finnish hospitals conducted prospective incidence surveillance for healthcare-associated BSIs 1999-2011. A common protocol for laboratory-based case finding was used and only BSIs with onset $>48 \mathrm{hrs}$ after admission were included. Patient-days with specialties were obtained from hospitals' information technology departments to calculate incidence densities (ID) with 95\% confidence intervals (CI). The ranking positions of hospitals were calculated for crude IDs and IDs adjusted by specialties and hospital type in mixed effect's negative binomial regression model. The effects in the model were considered to be constant over selected time period. The agreement and the correlation between rankings of the IDs were assessed by Cohen's kappa and Spearman's correlation coefficient, respectively.

\section{Results}

We identified 7855 BSIs of which 990 were SA-BSIs. For all BSIs, IDs varied from 0.16 per 1,000 patient-days to
0.79 between hospitals and for SA-BSIs from 0.03 to 0.10 . There were clear differences in crude and adjusted ranking positions of hospitals, but CIs were wide and mostly overlapped. The agreement between adjusted rankings was more fair than with crude ranking with its adjusted counterpart, kappa $0.3(\mathrm{p}=0.018)$ vs. 0.2 $(\mathrm{p}=0.078)$. Agreement of the two crude rankings was 0.1 $(\mathrm{p}=0.63)$. Correlation coefficients were 0.81 for adjusted BSI and SA-BSI rankings, and 0.69 for crude rankings.

\section{Conclusion}

Both the overall BSI ranking and SA-BSI ranking identified outliers. Adjusting by specialties and hospital type may be needed when ranking overall BSI rates but not for SA-BSI rates. SA-BSIs can be a useful indicator for hospital performance, stimulating the use of surveillance data. However, the rankings must be interpreted with caution, especially when numbers are small during a short period of surveillance.

\section{Disclosure of interest}

None declared.

Published: 20 June 2013

doi:10.1186/2047-2994-2-S1-063

Cite this article as: Kärki et al:: 0063: Healthcare-associated bloodstream infections in Finland, 1999-2011 - adjusted ranking of hospitals by Staphylococcus aureus rates. Antimicrobial Resistance and Infection Control 2013 2(Suppl 1):063.

National Institute for Health and Welfare, Helsinki, Finland

(c) 2013 Kärki et al; licensee BioMed Central Ltd. This is an Open Access article distributed under the terms of the Creative Commons 